\title{
Publisher Correction: EphA2 is an epithelial cell pattern recognition receptor for fungal $\beta$-glucans
}

Marc Swidergall (D), Norma V. Solis, Michail S. Lionakis and Scott G. Filler (D)

Correction to: Nature Microbiology https://doi.org/10.1038/s41564-017-0059-5, published online 13 November 2017.

In the version of this Article originally published, technical problems led to errors in Figs. $2 \mathrm{~d}$ and $5 \mathrm{~b}$. In the western blot panel in Fig. 2d, actin bands were misaligned at the bottom of the image and did not line up with the other bands in the panel; the corrected figure is shown below. In Fig. 5b, the upper right panel had an incorrect title of 'CXCL3/KC'; it should have instead been 'CXCL1/KC'. These errors have now been corrected in all versions of the Article.

Published online: 13 February 2018

https://doi.org/10.1038/s41564-017-0100-8
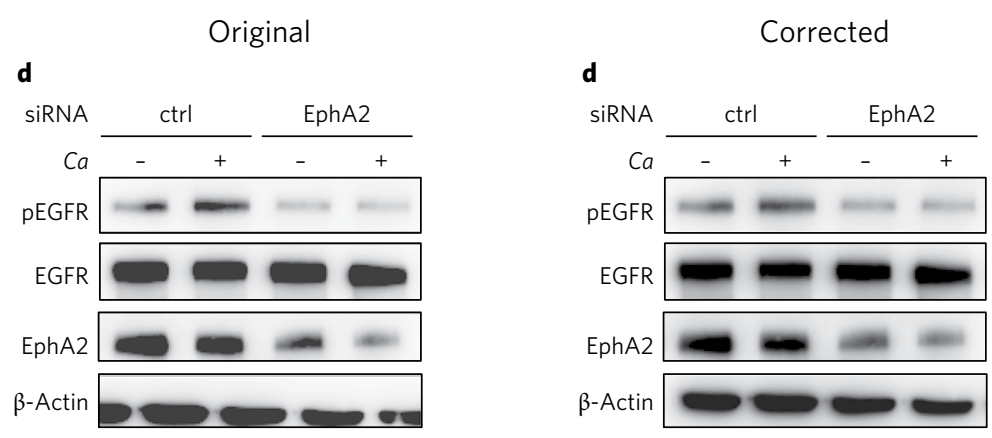

Fig. 2d | Original and corrected panels. 